\title{
Statistische Wählerstromananalyse der Nationalratswahl 2002
}

\author{
Erich Neuwirth \\ Institut für Statistik und Decision Support Systems \\ Universität Wien
}

\begin{abstract}
Zusammenfassung: Wir berechnen statistische Schätzungen für die Wählerströme zwischen den einzelnen Parteien bei der Nationalratswahl 2002 vom 24. November 2002. Diese Wählerströme werden für die einzelnen Bundesländer berechnet und dabei zeigen sich erhebliche regionale Unterschiede im Wahlverhalten. Zusätzlich stellen wir die regionalen Stimmenbilanzen der Parteien in grauwertcodierten Landkarten dar. Dabei lassen sich ebenfalls interessante Unterschiede feststellen.
\end{abstract}

\begin{abstract}
We present statistical estimations for voter transition rates between the political parties at the Austrian Parliament elections of November 24, 2002. The analysis is performed on the province level, and thereby rather noticeable differences in voting behavior are discovered. Additionally, we graph regional party wins or losses in grey-value-shaded maps, allowing us to investigate interesting regional differences.
\end{abstract}

Schlüsselwörter: Wahlanalyse, Wählerstromanalyse.

\section{Die wichtigsten Ergebnisse}

- Die mit Abstand größte Wählerbewegung war ein direkter Übergang von der FPÖ zur ÖVP im Umfang von mehr als 630.000 Stimmen. Diese direkten Verluste waren in Stimmen ausgedrückt in der Steiermark am größten.

- Die zweitstärkste Wählerbewegung war die Mobilisierung von mehr als 130.000 früheren Nichtwählern, die diesmal SPÖ gewählt haben. Diese Wählerbewegung war in Niederösterreich am größten.

- Die ÖVP konnte ebenfalls fast 100.000 an früheren Nichtwählern für sich mobilisieren, und zwar hauptsächlich in Tirol, Vorarlberg und Wien.

- Nur in Wien konnte die SPÖ relativ viele, nämlich ca. 70.000 frühere FPÖ-Wähler für sich gewinnen.

- Das Liberale Forum hat fast 130.000 seiner früheren Wähler an die Grünen verloren.

- Die Grünen erzielten den größten Teil ihrer Gewinne auf Kosten des Liberalen Forums, zum geringeren Teil aus der Mobilisierung früherer Nichtwähler. Sie verloren aber in einer schon merkbaren Größenordnung an die ÖVP und in geringerem Umfang an die SPÖ.

- Insgesamt haben ca. 1,4 Millionen Wahlberechtigte eine andere Wahlentscheidung als bei der Nationalratswahl 1999 getroffen. 


\section{Erste Übersichten und Grafische Darstellungen}

Basis aller unserer Untersuchungen sind die Anteile der Wähler der 5 Parteien SPÖ, ÖVP, FPÖ, Grüne und LIF bei den Nationalratswahlen 1999 und 2002. Bei der Nationalratswahl 2002 wurde auch die KPÖ getrennt ausgewiesen.

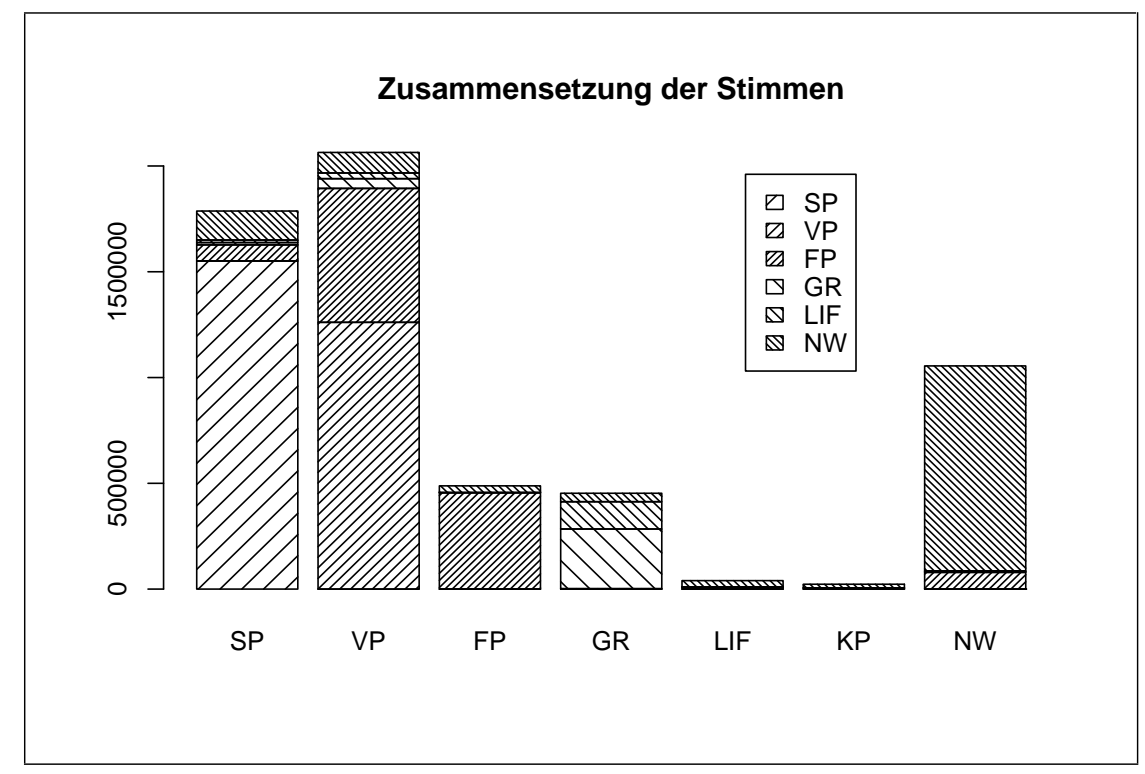

Diese Grafik ist folgendermaßen zu interpretieren:

Der erste Balken dieser Grafik repräsentiert die SPÖ-Stimmen von 1999. Die Unterteilung dieses Balkens gibt an, wie sich die SPÖ-Wähler von 1999 bei der Wahl 2002 auf sämtliche kandidierenden Parteien verteilt haben. Da die SPÖ praktisch alle ihre Wähler von 1999 halten konnte, ist dieser Balken nicht weiter unterteilt. Deutlich sichtbar ist, dass die FPÖ mehr als die Hälfte ihrer Wähler von 1999 an die ÖVP verloren hat.

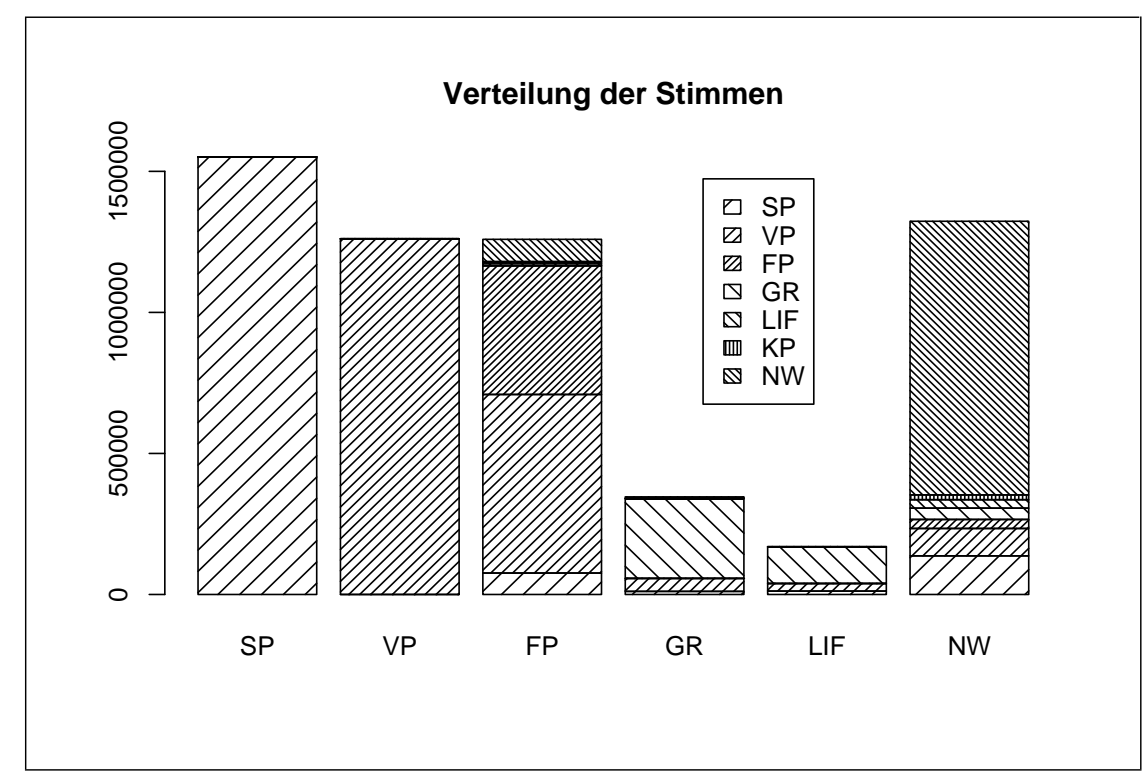

Diese Grafik veranschaulicht die Zusammensetzung der Stimmen der einzelnen Parteien. 
Der erste Balken dieser Grafik stellt die Zusammensetzung der SPÖ-Stimmen von 2002 dar. Man sieht ganz deutlich, dass die SPÖ zu 90\% hauptsächlich von Wählern gewählt wurde, die sie auch schon bei der NRW 1999 gewählt haben. Hinzugekommen sind frühere Nichtwähler und in geringem Umfang auch frühere FPÖ-Wähler.

\section{Landkarten}

Die folgenden Landkarten stellen die Anteilsveränderungen der einzelnen Parteien in den politischen Bezirken in Prozent der gültigen Stimmen dar.
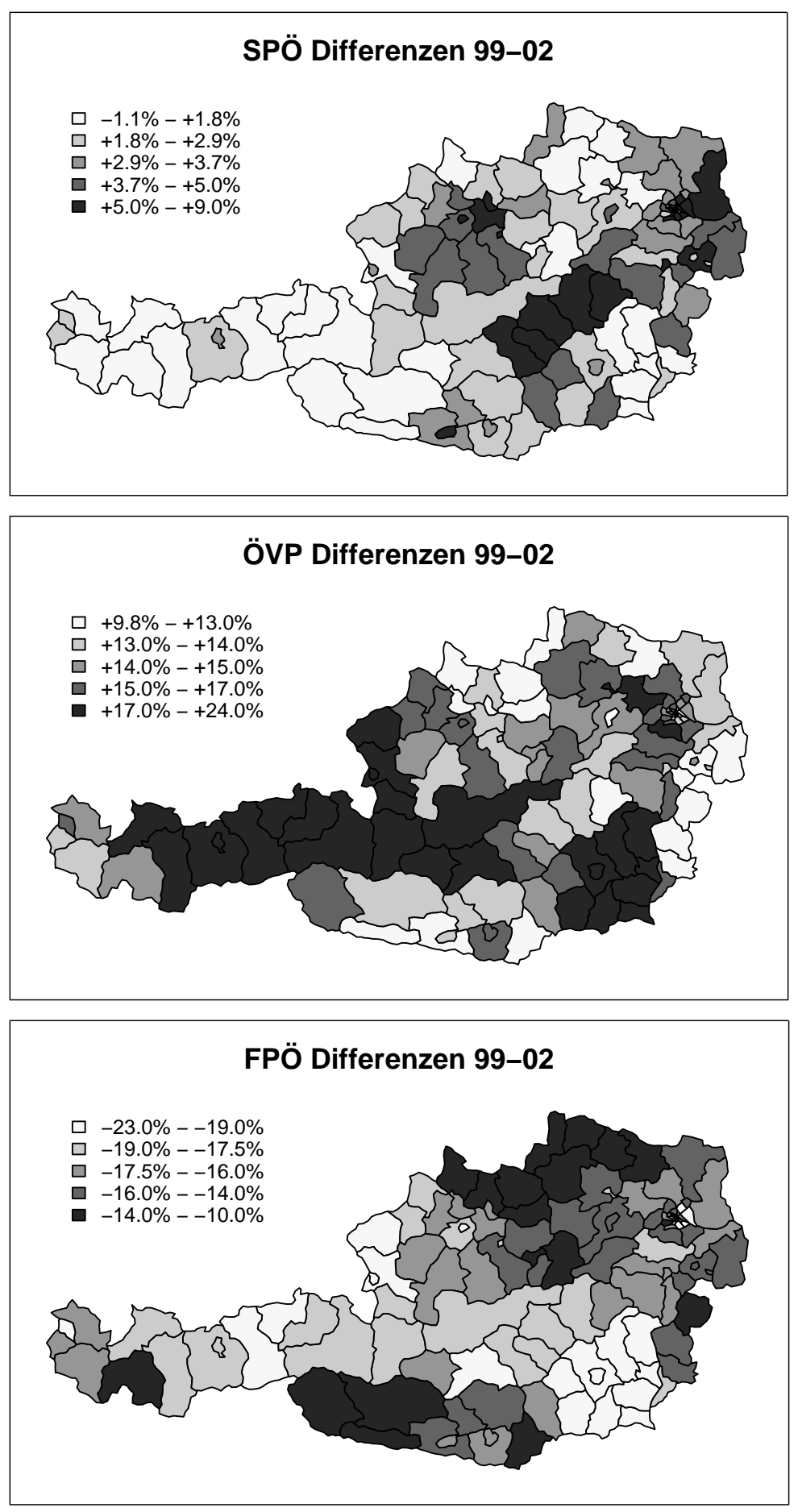


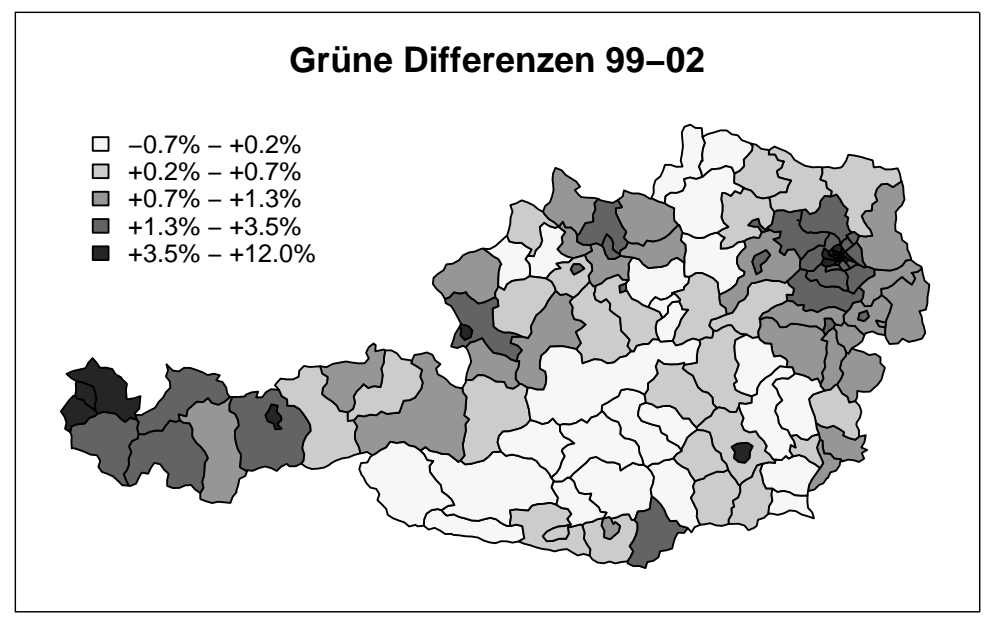

\section{Tabellenteil}

\subsection{Wählerbewegungen in Stimmen bundesweit}

\begin{tabular}{|l|r|r|r|r|r|r|r|r|}
\hline & SPÖ & ÖVP & FPÖ & GR & LIF & KPÖ & NW+So & Schw. \\
\hline SPÖ & 1551000 & 0 & 0 & 0 & 0 & 0 & 0 & 36000 \\
\hline ÖVP & 0 & 1261000 & 0 & 0 & 0 & 0 & 0 & 10000 \\
\hline FPÖ & 76000 & 633000 & 456000 & 2000 & 8000 & 5000 & 79000 & 44000 \\
\hline GR & 11000 & 46000 & 0 & 282000 & 1000 & 1000 & 5000 & 21000 \\
\hline LIF & 12000 & 27000 & 0 & 129000 & 1000 & 0 & 1000 & 22000 \\
\hline NW+So & 137000 & 97000 & 32000 & 40000 & 30000 & 17000 & 970000 & 13000 \\
\hline
\end{tabular}

Wie ist diese Tabelle zu lesen (ein Beispiel):

Die erste Zeile bedeutet, dass praktisch alle Wähler, die 1999 die SPÖ oder die ÖVP gewählt haben, auch 2002 wieder dieselbe Partei gewählt haben. 76.000 FPÖ-Wähler von 1999 sind 2002 zur SPÖ gewechselt, und 633.000 FPÖ-Wähler von 1999 sind 2002 zur ÖVP gewechselt. Die ausgewiesenen Zahlen sind mit einer statistischen Schwankungsbreite, die für jede Partei getrennt in der letzten Spalte ausgewiesen wird, behaftet.

\subsection{Saldierte Wählerströme bundesweit}

\begin{tabular}{|l|r|r|r|r|r|r|r|}
\hline & SPÖ & ÖVP & FPÖ & GR & LIF & KPÖ & NW+So \\
\hline SPÖ & 1551000 & 0 & 76000 & 11000 & 12000 & 0 & 137000 \\
\hline ÖVP & 0 & 1261000 & 633000 & 46000 & 27000 & 0 & 97000 \\
\hline FPÖ & -76000 & -633000 & 456000 & -2000 & -8000 & -5000 & -47000 \\
\hline GR & -11000 & -46000 & 2000 & 282000 & 128000 & -1000 & 35000 \\
\hline LIF & -12000 & -27000 & 8000 & -128000 & 1000 & 0 & 29000 \\
\hline KPÖ & 0 & 0 & 5000 & 1000 & 0 & & 17000 \\
\hline NW+So & -137000 & -97000 & 47000 & -35000 & -29000 & -17000 & 0 \\
\hline
\end{tabular}

Wie ist diese Tabelle zu lesen (ein Beispiel):

Die erste Zeile bedeutet, dass die SPÖ etwa 1.551.000 Wähler von 1999 ,halten konnte“. Die Stimmenbilanz gegenüber der FPÖ beträgt +76.000 Stimmen, die SPÖ hat also 76.000 Stimmen mehr von der FPÖ gewonnen als sie an die FPÖ verloren hat (da sie, 
siehe vorige Tabelle, überhaupt keine Stimmen an die FPÖ verloren hat, ist in diesem speziellen Fall Nettobilanz gleich Bruttobilanz).

Da unsere Analyse die Ergebnisse der KPÖ 1999 nicht berücksichtigt, ist die entsprechende Zelle der Tabelle leer.

\subsection{Wählerbewegungen in Prozent der Ausgangspartei bundesweit}

\begin{tabular}{|l|r|r|r|r|r|r|r|r|}
\hline & SPÖ & ÖVP & FPÖ & GR & LIF & KPÖ & NW+So & Schw. \\
\hline SPÖ & $100,0 \%$ & $0,0 \%$ & $0,0 \%$ & $0,0 \%$ & $0,0 \%$ & $0,0 \%$ & $0,0 \%$ & $2,3 \%$ \\
\hline ÖVP & $0,0 \%$ & $100,0 \%$ & $0,0 \%$ & $0,0 \%$ & $0,0 \%$ & $0,0 \%$ & $0,0 \%$ & $0,8 \%$ \\
\hline FPÖ & $6,1 \%$ & $50,3 \%$ & $36,2 \%$ & $0,2 \%$ & $0,6 \%$ & $0,4 \%$ & $6,3 \%$ & $3,5 \%$ \\
\hline GR & $3,2 \%$ & $13,3 \%$ & $0,0 \%$ & $81,4 \%$ & $0,3 \%$ & $0,4 \%$ & $1,4 \%$ & $5,9 \%$ \\
\hline LIF & $7,0 \%$ & $15,9 \%$ & $0,0 \%$ & $75,7 \%$ & $0,7 \%$ & $0,0 \%$ & $0,7 \%$ & $12,7 \%$ \\
\hline NW+So & $10,3 \%$ & $7,3 \%$ & $2,4 \%$ & $3,1 \%$ & $2,3 \%$ & $1,3 \%$ & $73,3 \%$ & $1,0 \%$ \\
\hline
\end{tabular}

Wie ist diese Tabelle zu lesen (ein Beispiel):

Die erste Zeile gibt an, wie sich die SPÖ-Wähler von 1999 bei der NRW 2002 anteilsmäßig auf die Parteien verteilt haben. Die SPÖ konnte 100\% ihrer Wähler halten. Die Grünen verloren ca. 13\% ihrer Wähler von 1999 an die ÖVP, was in der reinen Nettostimmenbilanz nicht unmittelbar ersichtlich ist.

\subsection{Zusammensetzung der Parteienstimmen bundesweit}

\begin{tabular}{|c|c|c|c|c|c|c|}
\hline & SPÖ & ÖVP & FPÖ & GR & LIF & NW+So \\
\hline SPÖ & $86,8 \%$ & $0,0 \%$ & $4,3 \%$ & $0,6 \%$ & $0,7 \%$ & $7,7 \%$ \\
\hline ÖVP & $0,0 \%$ & $61,1 \%$ & $30,7 \%$ & $2,2 \%$ & $1,3 \%$ & $4,7 \%$ \\
\hline FPÖ & $0,0 \%$ & $0,0 \%$ & $93,5 \%$ & $0,0 \%$ & $0,0 \%$ & $6,5 \%$ \\
\hline GR & $0,0 \%$ & $0,0 \%$ & $0,4 \%$ & $62,2 \%$ & $28,4 \%$ & $8,9 \%$ \\
\hline LIF & $0,0 \%$ & $0,0 \%$ & $20,0 \%$ & $2,8 \%$ & $2,8 \%$ & $74,5 \%$ \\
\hline NW+So & $0,0 \%$ & $0,0 \%$ & $22,4 \%$ & $6,0 \%$ & $0,0 \%$ & $71,6 \%$ \\
\hline
\end{tabular}

Wie ist diese Tabelle zu lesen (ein Beispiel): 86,8\% der SPÖ-Wähler von 2002 haben schon 1999 die SPÖ gewählt, 4,3\% der SPÖ-Wähler haben 1999 die FPÖ gewählt.

\subsection{Wählerbewegungen in Prozent der Wahlberechtigten bundesweit}

\begin{tabular}{|c|c|c|c|c|c|c|c|c|}
\hline & SPÖ & ÖVP & FPÖ & GR & LIF & KPÖ & NW+So & Schw. \\
\hline SPÖ & $26,2 \%$ & $0,0 \%$ & $0,0 \%$ & $0,0 \%$ & $0,0 \%$ & $0,0 \%$ & $0,0 \%$ & $0,6 \%$ \\
\hline ÖVP & $0,0 \%$ & $21,3 \%$ & $0,0 \%$ & $0,0 \%$ & $0,0 \%$ & $0,0 \%$ & $0,0 \%$ & $0,2 \%$ \\
\hline FPÖ & $1,3 \%$ & $10,7 \%$ & $7,7 \%$ & $0,0 \%$ & $0,1 \%$ & $0,1 \%$ & $1,3 \%$ & $0,7 \%$ \\
\hline GR & $0,2 \%$ & $0,8 \%$ & $0,0 \%$ & $4,8 \%$ & $0,0 \%$ & $0,0 \%$ & $0,1 \%$ & $0,3 \%$ \\
\hline LIF & $0,2 \%$ & $0,5 \%$ & $0,0 \%$ & $2,2 \%$ & $0,0 \%$ & $0,0 \%$ & $0,0 \%$ & $0,4 \%$ \\
\hline NW+So & $2,3 \%$ & $1,6 \%$ & $0,5 \%$ & $0,7 \%$ & $0,5 \%$ & $0,3 \%$ & $16,4 \%$ & $0,2 \%$ \\
\hline
\end{tabular}

Wie ist diese Tabelle zu lesen (ein Beispiel): 26,2\% der Wahlberechtigten haben 1999 und 2002 die SPÖ gewählt, von der FPÖ zur SPÖ gewechselt sind 1,3\% der Wahlberechtigten. 


\subsection{Wählerbewegungen in Stimmen - bundesländerweise}

\subsubsection{SPÖ-Wähler von 1999}

\begin{tabular}{|c|c|c|c|c|c|c|c|c|}
\hline & SPÖ & ÖVP & FPÖ & GR & LIF & KPÖ & NW+So & Schw. \\
\hline B & 78000 & 0 & 0 & 0 & 0 & 0 & 0 & 2000 \\
\hline K & 120000 & 0 & 0 & 0 & 0 & 0 & 0 & 3000 \\
\hline N & 330000 & 0 & 0 & 0 & 0 & 0 & 0 & 5000 \\
\hline $\mathrm{O}$ & 273000 & 0 & 0 & 0 & 0 & 0 & 0 & 4000 \\
\hline Sa & 81000 & 0 & 0 & 0 & 0 & 0 & 0 & 2000 \\
\hline St & 246000 & 0 & 0 & 0 & 0 & 0 & 0 & 3000 \\
\hline T & 85000 & 0 & 0 & 0 & 0 & 0 & 0 & 2000 \\
\hline $\mathrm{V}$ & 32000 & 0 & 0 & 0 & 0 & 0 & 0 & 2000 \\
\hline W & 306000 & 0 & 0 & 0 & 0 & 0 & 0 & 35000 \\
\hline
\end{tabular}

\subsection{2 ÖVP-Wähler von 1999}

\begin{tabular}{|c|c|c|c|c|c|c|c|c|}
\hline & SPÖ & ÖVP & FPÖ & GR & LIF & KPO & NW+So & Schw. \\
\hline $\mathrm{B}$ & 0 & 57000 & 0 & 0 & 0 & 0 & 0 & 1000 \\
\hline $\mathrm{K}$ & 0 & 54000 & 0 & 0 & 0 & 0 & 0 & 2000 \\
\hline $\mathrm{N}$ & 0 & 321000 & 0 & 0 & 0 & 0 & 0 & 3000 \\
\hline $\mathrm{O}$ & 0 & 236000 & 0 & 0 & 0 & 0 & 0 & 2000 \\
\hline $\mathrm{Sa}$ & 0 & 78000 & 0 & 0 & 0 & 0 & 0 & 2000 \\
\hline $\mathrm{St}$ & 0 & 195000 & 0 & 0 & 0 & 0 & 0 & 3000 \\
\hline $\mathrm{T}$ & 0 & 121000 & 0 & 0 & 0 & 0 & 0 & 2000 \\
\hline $\mathrm{V}$ & 0 & 61000 & 0 & 0 & 0 & 0 & 0 & 2000 \\
\hline $\mathrm{W}$ & 0 & 137000 & 0 & 0 & 0 & 0 & 0 & 7000 \\
\hline
\end{tabular}

\subsubsection{FPÖ-Wähler von 1999}

\begin{tabular}{|c|c|c|c|c|c|c|c|c|}
\hline & SPÖ & ÖVP & FPO & GR & LIF & KPO & NW+So & Schw. \\
\hline $\mathrm{B}$ & 1000 & 19000 & 12000 & 1000 & 1000 & 0 & 5000 & 2000 \\
\hline $\mathrm{K}$ & 3000 & 30000 & 83000 & 0 & 1000 & 1000 & 12000 & 5000 \\
\hline $\mathrm{N}$ & 0 & 137000 & 63000 & 0 & 1000 & 1000 & 17000 & 7000 \\
\hline $\mathrm{O}$ & 0 & 109000 & 90000 & 0 & 0 & 1000 & 22000 & 5000 \\
\hline $\mathrm{Sa}$ & 0 & 54000 & 29000 & 0 & 0 & 0 & 0 & 4000 \\
\hline $\mathrm{St}$ & 0 & 143000 & 66000 & 0 & 0 & 0 & 4000 & 6000 \\
\hline $\mathrm{T}$ & 1000 & 52000 & 37000 & 0 & 4000 & 1000 & 8000 & 3000 \\
\hline $\mathrm{V}$ & 1000 & 11000 & 26000 & 1000 & 2000 & 1000 & 10000 & 3000 \\
\hline $\mathrm{W}$ & 71000 & 79000 & 51000 & 0 & 0 & 0 & 0 & 42000 \\
\hline
\end{tabular}




\subsubsection{Grüne-Wähler von 1999}

\begin{tabular}{|c|c|c|c|c|c|c|c|c|}
\hline & SPÖ & ÖVP & FPÖ & GR & LIF & KPÖ & NW+So & Schw. \\
\hline B & 0 & 1000 & 0 & 6000 & 0 & 0 & 0 & 2000 \\
\hline K & 0 & 7000 & 0 & 11000 & 0 & 0 & 0 & 3000 \\
\hline N & 0 & 18000 & 0 & 40000 & 0 & 0 & 0 & 5000 \\
\hline O & 1000 & 11000 & 0 & 49000 & 1000 & 0 & 0 & 5000 \\
\hline Sa & 1000 & 8000 & 0 & 15000 & 0 & 0 & 0 & 4000 \\
\hline St & 0 & 2000 & 0 & 33000 & 1000 & 1000 & 5000 & 5000 \\
\hline T & 8000 & 0 & 0 & 27000 & 0 & 0 & 0 & 4000 \\
\hline V & 1000 & 0 & 0 & 17000 & 0 & 0 & 0 & 3000 \\
\hline W & 0 & 0 & 0 & 83000 & 0 & 0 & 0 & 17000 \\
\hline
\end{tabular}

\subsubsection{LIF-Wähler von 1999}

\begin{tabular}{|c|c|c|c|c|c|c|c|c|}
\hline & SPÖ & ÖVP & FPO & GR & LIF & KPÖ & NW+So & Schw. \\
\hline $\mathrm{B}$ & 1000 & 1000 & 0 & 1000 & 0 & 0 & 0 & 1000 \\
\hline $\mathrm{K}$ & 0 & 1000 & 0 & 6000 & 0 & 0 & 1000 & 1000 \\
\hline $\mathrm{N}$ & 0 & 2000 & 0 & 25000 & 1000 & 0 & 0 & 4000 \\
\hline $\mathrm{O}$ & 7000 & 0 & 0 & 15000 & 0 & 0 & 0 & 4000 \\
\hline $\mathrm{Sa}$ & 0 & 0 & 0 & 11000 & 0 & 0 & 0 & 4000 \\
\hline $\mathrm{St}$ & 0 & 0 & 0 & 19000 & 0 & 0 & 0 & 4000 \\
\hline $\mathrm{T}$ & 0 & 0 & 0 & 14000 & 0 & 0 & 0 & 3000 \\
\hline $\mathrm{V}$ & 3000 & 0 & 0 & 5000 & 0 & 0 & 0 & 3000 \\
\hline $\mathrm{W}$ & 0 & 23000 & 0 & 33000 & 0 & 0 & 0 & 19000 \\
\hline
\end{tabular}

\subsubsection{Nichtwähler und sonstige Wähler von 1999}

\begin{tabular}{|c|c|c|c|c|c|c|c|c|}
\hline & SPÖ & ÖVP & FPÖ & GR & LIF & KPÖ & NW+So & Schw. \\
\hline B & 7000 & 3000 & 0 & 0 & 0 & 0 & 22000 & 2000 \\
\hline K & 9000 & 11000 & 0 & 3000 & 2000 & 1000 & 69000 & 4000 \\
\hline N & 38000 & 8000 & 6000 & 6000 & 5000 & 3000 & 134000 & 6000 \\
\hline O & 32000 & 7000 & 0 & 8000 & 5000 & 3000 & 138000 & 5000 \\
\hline Sa & 8000 & 0 & 3000 & 4000 & 3000 & 1000 & 66000 & 3000 \\
\hline St & 32000 & 0 & 7000 & 0 & 5000 & 5000 & 156000 & 5000 \\
\hline T & 2000 & 25000 & 2000 & 4000 & 2000 & 1000 & 86000 & 3000 \\
\hline V & 3000 & 21000 & 0 & 5000 & 1000 & 0 & 36000 & 4000 \\
\hline W & 6000 & 22000 & 14000 & 11000 & 8000 & 4000 & 263000 & 4000 \\
\hline
\end{tabular}




\subsection{Wählerbewegungen in Prozent der Ausgangspartei - bundesländerweise}

\subsubsection{SPÖ-Wähler von 1999}

\begin{tabular}{|c|c|c|c|c|c|c|c|c|}
\hline & SPÖ & ÖVP & FPÖ & GR & LIF & KPÖ & NW+So & Schw. \\
\hline B & $100,0 \%$ & $0,0 \%$ & $0,0 \%$ & $0,0 \%$ & $0,0 \%$ & $0,0 \%$ & $0,0 \%$ & $1,9 \%$ \\
\hline K & $100,0 \%$ & $0,0 \%$ & $0,0 \%$ & $0,0 \%$ & $0,0 \%$ & $0,0 \%$ & $0,0 \%$ & $2,5 \%$ \\
\hline N & $100,0 \%$ & $0,0 \%$ & $0,0 \%$ & $0,0 \%$ & $0,0 \%$ & $0,0 \%$ & $0,0 \%$ & $1,4 \%$ \\
\hline O & $100,0 \%$ & $0,0 \%$ & $0,0 \%$ & $0,0 \%$ & $0,0 \%$ & $0,0 \%$ & $0,0 \%$ & $1,4 \%$ \\
\hline Sa & $100,0 \%$ & $0,0 \%$ & $0,0 \%$ & $0,0 \%$ & $0,0 \%$ & $0,0 \%$ & $0,0 \%$ & $2,8 \%$ \\
\hline St & $100,0 \%$ & $0,0 \%$ & $0,0 \%$ & $0,0 \%$ & $0,0 \%$ & $0,0 \%$ & $0,0 \%$ & $1,1 \%$ \\
\hline T & $100,0 \%$ & $0,0 \%$ & $0,0 \%$ & $0,0 \%$ & $0,0 \%$ & $0,0 \%$ & $0,0 \%$ & $2,8 \%$ \\
\hline V & $100,0 \%$ & $0,0 \%$ & $0,0 \%$ & $0,0 \%$ & $0,0 \%$ & $0,0 \%$ & $0,0 \%$ & $5,4 \%$ \\
\hline W & $100,0 \%$ & $0,0 \%$ & $0,0 \%$ & $0,0 \%$ & $0,0 \%$ & $0,0 \%$ & $0,0 \%$ & $11,5 \%$ \\
\hline
\end{tabular}

\subsection{2 ÖVP-Wähler von 1999}

\begin{tabular}{|c|c|c|c|c|c|c|c|c|}
\hline & SPÖ & ÖVP & FPÖ & GR & LIF & KPÖ & NW+So & Schw. \\
\hline B & $0,0 \%$ & $100,0 \%$ & $0,0 \%$ & $0,0 \%$ & $0,0 \%$ & $0,0 \%$ & $0,0 \%$ & $2,1 \%$ \\
\hline K & $0,0 \%$ & $99,2 \%$ & $0,0 \%$ & $0,0 \%$ & $0,0 \%$ & $0,0 \%$ & $0,8 \%$ & $3,7 \%$ \\
\hline $\mathrm{N}$ & $0,0 \%$ & $100,0 \%$ & $0,0 \%$ & $0,0 \%$ & $0,0 \%$ & $0,0 \%$ & $0,0 \%$ & $0,9 \%$ \\
\hline $\mathrm{O}$ & $0,0 \%$ & $100,0 \%$ & $0,0 \%$ & $0,0 \%$ & $0,0 \%$ & $0,0 \%$ & $0,0 \%$ & $0,9 \%$ \\
\hline $\mathrm{Sa}$ & $0,0 \%$ & $100,0 \%$ & $0,0 \%$ & $0,0 \%$ & $0,0 \%$ & $0,0 \%$ & $0,0 \%$ & $2,2 \%$ \\
\hline $\mathrm{St}$ & $0,0 \%$ & $100,0 \%$ & $0,0 \%$ & $0,0 \%$ & $0,0 \%$ & $0,0 \%$ & $0,0 \%$ & $1,4 \%$ \\
\hline $\mathrm{T}$ & $0,0 \%$ & $100,0 \%$ & $0,0 \%$ & $0,0 \%$ & $0,0 \%$ & $0,0 \%$ & $0,0 \%$ & $1,4 \%$ \\
\hline $\mathrm{V}$ & $0,0 \%$ & $100,0 \%$ & $0,0 \%$ & $0,0 \%$ & $0,0 \%$ & $0,0 \%$ & $0,0 \%$ & $2,9 \%$ \\
\hline $\mathrm{W}$ & $0,0 \%$ & $100,0 \%$ & $0,0 \%$ & $0,0 \%$ & $0,0 \%$ & $0,0 \%$ & $0,0 \%$ & $5,4 \%$ \\
\hline
\end{tabular}

\subsubsection{FPÖ-Wähler von 1999}

\begin{tabular}{|c|c|c|c|c|c|c|c|c|}
\hline & SPÖ & ÖVP & FPÖ & GR & LIF & KPÖ & NW+So & Schw. \\
\hline B & $3,2 \%$ & $48,3 \%$ & $31,1 \%$ & $2,3 \%$ & $2,1 \%$ & $1,2 \%$ & $11,7 \%$ & $4,9 \%$ \\
\hline K & $2,1 \%$ & $22,9 \%$ & $63,9 \%$ & $0,0 \%$ & $0,5 \%$ & $0,9 \%$ & $9,6 \%$ & $3,6 \%$ \\
\hline N & $0,0 \%$ & $62,4 \%$ & $28,7 \%$ & $0,0 \%$ & $0,3 \%$ & $0,6 \%$ & $8,0 \%$ & $3,2 \%$ \\
\hline O & $0,0 \%$ & $49,3 \%$ & $40,5 \%$ & $0,0 \%$ & $0,0 \%$ & $0,2 \%$ & $9,9 \%$ & $2,1 \%$ \\
\hline Sa & $0,0 \%$ & $65,0 \%$ & $35,0 \%$ & $0,0 \%$ & $0,0 \%$ & $0,0 \%$ & $0,0 \%$ & $4,8 \%$ \\
\hline St & $0,0 \%$ & $67,1 \%$ & $30,9 \%$ & $0,0 \%$ & $0,0 \%$ & $0,0 \%$ & $2,0 \%$ & $2,7 \%$ \\
\hline T & $0,7 \%$ & $50,8 \%$ & $35,9 \%$ & $0,0 \%$ & $3,5 \%$ & $1,0 \%$ & $8,1 \%$ & $3,1 \%$ \\
\hline V & $2,3 \%$ & $21,8 \%$ & $49,2 \%$ & $2,1 \%$ & $4,3 \%$ & $1,4 \%$ & $18,9 \%$ & $5,2 \%$ \\
\hline W & $35,2 \%$ & $39,3 \%$ & $25,4 \%$ & $0,0 \%$ & $0,0 \%$ & $0,0 \%$ & $0,0 \%$ & $21,1 \%$ \\
\hline
\end{tabular}




\subsubsection{Grüne-Wähler von 1999}

\begin{tabular}{|c|c|c|c|c|c|c|c|c|}
\hline & SPÖ & ÖVP & FPÖ & GR & LIF & KPÖ & NW+So & Schw. \\
\hline $\mathrm{B}$ & $0,0 \%$ & $8,3 \%$ & $0,0 \%$ & $91,7 \%$ & $0,0 \%$ & $0,0 \%$ & $0,0 \%$ & $23,7 \%$ \\
\hline $\mathrm{K}$ & $0,0 \%$ & $40,9 \%$ & $0,0 \%$ & $59,1 \%$ & $0,0 \%$ & $0,0 \%$ & $0,0 \%$ & $14,9 \%$ \\
\hline $\mathrm{N}$ & $0,0 \%$ & $30,5 \%$ & $0,0 \%$ & $69,5 \%$ & $0,0 \%$ & $0,0 \%$ & $0,0 \%$ & $8,8 \%$ \\
\hline $\mathrm{O}$ & $1,3 \%$ & $17,4 \%$ & $0,0 \%$ & $80,3 \%$ & $0,9 \%$ & $0,0 \%$ & $0,0 \%$ & $8,5 \%$ \\
\hline $\mathrm{Sa}$ & $4,7 \%$ & $32,4 \%$ & $0,0 \%$ & $62,9 \%$ & $0,0 \%$ & $0,0 \%$ & $0,0 \%$ & $16,6 \%$ \\
\hline $\mathrm{St}$ & $0,0 \%$ & $5,3 \%$ & $0,0 \%$ & $78,9 \%$ & $1,3 \%$ & $3,4 \%$ & $11,1 \%$ & $11,8 \%$ \\
\hline $\mathrm{T}$ & $23,5 \%$ & $0,0 \%$ & $0,0 \%$ & $76,5 \%$ & $0,0 \%$ & $0,0 \%$ & $0,0 \%$ & $10,4 \%$ \\
\hline $\mathrm{V}$ & $3,7 \%$ & $0,0 \%$ & $0,0 \%$ & $96,3 \%$ & $0,0 \%$ & $0,0 \%$ & $0,0 \%$ & $17,1 \%$ \\
\hline $\mathrm{W}$ & $0,0 \%$ & $0,0 \%$ & $0,0 \%$ & $100,0 \%$ & $0,0 \%$ & $0,0 \%$ & $0,0 \%$ & $20,7 \%$ \\
\hline
\end{tabular}

\subsubsection{LIF-Wähler von 1999}

\begin{tabular}{|c|c|c|c|c|c|c|c|c|}
\hline & SPÖ & ÖVP & FPÖ & GR & LIF & KPÖ & NW+So & Schw. \\
\hline B & $27,7 \%$ & $29,2 \%$ & $0,0 \%$ & $43,2 \%$ & $0,0 \%$ & $0,0 \%$ & $0,0 \%$ & $43,2 \%$ \\
\hline K & $4,6 \%$ & $7,3 \%$ & $0,0 \%$ & $73,6 \%$ & $0,0 \%$ & $0,0 \%$ & $14,6 \%$ & $15,2 \%$ \\
\hline $\mathrm{N}$ & $0,0 \%$ & $7,2 \%$ & $0,0 \%$ & $88,8 \%$ & $4,0 \%$ & $0,0 \%$ & $0,0 \%$ & $13,1 \%$ \\
\hline $\mathrm{O}$ & $33,1 \%$ & $0,0 \%$ & $0,0 \%$ & $66,9 \%$ & $0,0 \%$ & $0,0 \%$ & $0,0 \%$ & $20,2 \%$ \\
\hline $\mathrm{Sa}$ & $0,0 \%$ & $0,0 \%$ & $0,0 \%$ & $100,0 \%$ & $0,0 \%$ & $0,0 \%$ & $0,0 \%$ & $37,7 \%$ \\
\hline $\mathrm{St}$ & $0,0 \%$ & $0,0 \%$ & $0,0 \%$ & $100,0 \%$ & $0,0 \%$ & $0,0 \%$ & $0,0 \%$ & $21,2 \%$ \\
\hline $\mathrm{T}$ & $0,0 \%$ & $0,0 \%$ & $0,0 \%$ & $100,0 \%$ & $0,0 \%$ & $0,0 \%$ & $0,0 \%$ & $22,8 \%$ \\
\hline $\mathrm{V}$ & $39,7 \%$ & $0,0 \%$ & $0,0 \%$ & $60,3 \%$ & $0,0 \%$ & $0,0 \%$ & $0,0 \%$ & $40,1 \%$ \\
\hline $\mathrm{W}$ & $0,0 \%$ & $41,7 \%$ & $0,0 \%$ & $58,3 \%$ & $0,0 \%$ & $0,0 \%$ & $0,0 \%$ & $34,4 \%$ \\
\hline
\end{tabular}

\subsubsection{Nichtwähler und sonstige Wähler von 1999}

\begin{tabular}{|c|c|c|c|c|c|c|c|c|}
\hline & SPÖ & ÖVP & FPÖ & GR & LIF & KPÖ & NW+So & Schw. \\
\hline $\mathrm{B}$ & $21,2 \%$ & $9,7 \%$ & $0,0 \%$ & $0,2 \%$ & $0,0 \%$ & $0,0 \%$ & $68,9 \%$ & $7,3 \%$ \\
\hline $\mathrm{K}$ & $9,5 \%$ & $11,7 \%$ & $0,0 \%$ & $2,9 \%$ & $1,9 \%$ & $0,6 \%$ & $73,4 \%$ & $4,8 \%$ \\
\hline $\mathrm{N}$ & $18,9 \%$ & $3,9 \%$ & $3,1 \%$ & $3,2 \%$ & $2,6 \%$ & $1,3 \%$ & $66,9 \%$ & $3,1 \%$ \\
\hline $\mathrm{O}$ & $16,5 \%$ & $3,7 \%$ & $0,0 \%$ & $4,0 \%$ & $2,8 \%$ & $1,5 \%$ & $71,6 \%$ & $2,4 \%$ \\
\hline $\mathrm{Sa}$ & $9,8 \%$ & $0,0 \%$ & $3,3 \%$ & $4,8 \%$ & $3,0 \%$ & $1,2 \%$ & $78,0 \%$ & $4,1 \%$ \\
\hline $\mathrm{St}$ & $15,5 \%$ & $0,0 \%$ & $3,3 \%$ & $0,0 \%$ & $2,4 \%$ & $2,5 \%$ & $76,3 \%$ & $2,4 \%$ \\
\hline $\mathrm{T}$ & $2,0 \%$ & $20,7 \%$ & $1,6 \%$ & $2,9 \%$ & $1,5 \%$ & $0,8 \%$ & $70,4 \%$ & $2,6 \%$ \\
\hline $\mathrm{V}$ & $4,5 \%$ & $32,2 \%$ & $0,0 \%$ & $6,9 \%$ & $1,1 \%$ & $0,0 \%$ & $55,3 \%$ & $5,8 \%$ \\
\hline $\mathrm{W}$ & $1,9 \%$ & $6,6 \%$ & $4,3 \%$ & $3,5 \%$ & $2,3 \%$ & $1,2 \%$ & $80,2 \%$ & $1,2 \%$ \\
\hline
\end{tabular}




\subsection{Wählerbewegungen in Prozent der Wahlberechtigten - bundesländerweise}

\subsubsection{SPÖ-Wähler von 1999}

\begin{tabular}{|c|c|c|c|c|c|c|c|c|}
\hline & SPÖ & ÖVP & FPO & GR & LIF & KPÖ & NW+So & Schw. \\
\hline B & $36,1 \%$ & $0,0 \%$ & $0,0 \%$ & $0,0 \%$ & $0,0 \%$ & $0,0 \%$ & $0,0 \%$ & $0,7 \%$ \\
\hline K & $28,2 \%$ & $0,0 \%$ & $0,0 \%$ & $0,0 \%$ & $0,0 \%$ & $0,0 \%$ & $0,0 \%$ & $0,7 \%$ \\
\hline $\mathrm{N}$ & $28,5 \%$ & $0,0 \%$ & $0,0 \%$ & $0,0 \%$ & $0,0 \%$ & $0,0 \%$ & $0,0 \%$ & $0,4 \%$ \\
\hline $\mathrm{O}$ & $27,1 \%$ & $0,0 \%$ & $0,0 \%$ & $0,0 \%$ & $0,0 \%$ & $0,0 \%$ & $0,0 \%$ & $0,4 \%$ \\
\hline $\mathrm{Sa}$ & $22,5 \%$ & $0,0 \%$ & $0,0 \%$ & $0,0 \%$ & $0,0 \%$ & $0,0 \%$ & $0,0 \%$ & $0,6 \%$ \\
\hline $\mathrm{St}$ & $26,7 \%$ & $0,0 \%$ & $0,0 \%$ & $0,0 \%$ & $0,0 \%$ & $0,0 \%$ & $0,0 \%$ & $0,3 \%$ \\
\hline $\mathrm{T}$ & $17,7 \%$ & $0,0 \%$ & $0,0 \%$ & $0,0 \%$ & $0,0 \%$ & $0,0 \%$ & $0,0 \%$ & $0,5 \%$ \\
\hline $\mathrm{V}$ & $13,3 \%$ & $0,0 \%$ & $0,0 \%$ & $0,0 \%$ & $0,0 \%$ & $0,0 \%$ & $0,0 \%$ & $0,7 \%$ \\
\hline $\mathrm{W}$ & $27,5 \%$ & $0,0 \%$ & $0,0 \%$ & $0,0 \%$ & $0,0 \%$ & $0,0 \%$ & $0,0 \%$ & $3,2 \%$ \\
\hline
\end{tabular}

\subsection{2 ÖVP-Wähler von 1999}

\begin{tabular}{|c|c|c|c|c|c|c|c|c|}
\hline & SPO & $\ddot{O V P}$ & FPO & GR & LIF & KPÖ & NW+So & Schw. \\
\hline B & $0,0 \%$ & $26,3 \%$ & $0,0 \%$ & $0,0 \%$ & $0,0 \%$ & $0,0 \%$ & $0,0 \%$ & $0,6 \%$ \\
\hline K & $0,0 \%$ & $12,7 \%$ & $0,0 \%$ & $0,0 \%$ & $0,0 \%$ & $0,0 \%$ & $0,1 \%$ & $0,5 \%$ \\
\hline N & $0,0 \%$ & $27,8 \%$ & $0,0 \%$ & $0,0 \%$ & $0,0 \%$ & $0,0 \%$ & $0,0 \%$ & $0,3 \%$ \\
\hline O & $0,0 \%$ & $23,5 \%$ & $0,0 \%$ & $0,0 \%$ & $0,0 \%$ & $0,0 \%$ & $0,0 \%$ & $0,2 \%$ \\
\hline Sa & $0,0 \%$ & $21,7 \%$ & $0,0 \%$ & $0,0 \%$ & $0,0 \%$ & $0,0 \%$ & $0,0 \%$ & $0,5 \%$ \\
\hline St & $0,0 \%$ & $21,2 \%$ & $0,0 \%$ & $0,0 \%$ & $0,0 \%$ & $0,0 \%$ & $0,0 \%$ & $0,3 \%$ \\
\hline T & $0,0 \%$ & $25,2 \%$ & $0,0 \%$ & $0,0 \%$ & $0,0 \%$ & $0,0 \%$ & $0,0 \%$ & $0,4 \%$ \\
\hline V & $0,0 \%$ & $25,9 \%$ & $0,0 \%$ & $0,0 \%$ & $0,0 \%$ & $0,0 \%$ & $0,0 \%$ & $0,7 \%$ \\
\hline W & $0,0 \%$ & $12,4 \%$ & $0,0 \%$ & $0,0 \%$ & $0,0 \%$ & $0,0 \%$ & $0,0 \%$ & $0,7 \%$ \\
\hline
\end{tabular}

\subsubsection{FPÖ-Wähler von 1999}

\begin{tabular}{|c|c|c|c|c|c|c|c|c|}
\hline & SPÖ & ÖVP & FPÖ & GR & LIF & KPÖ & NW+So & Schw. \\
\hline B & $0,6 \%$ & $8,8 \%$ & $5,6 \%$ & $0,4 \%$ & $0,4 \%$ & $0,2 \%$ & $2,1 \%$ & $0,9 \%$ \\
\hline K & $0,6 \%$ & $7,0 \%$ & $19,5 \%$ & $0,0 \%$ & $0,2 \%$ & $0,3 \%$ & $2,9 \%$ & $1,1 \%$ \\
\hline N & $0,0 \%$ & $11,8 \%$ & $5,4 \%$ & $0,0 \%$ & $0,0 \%$ & $0,1 \%$ & $1,5 \%$ & $0,6 \%$ \\
\hline O & $0,0 \%$ & $10,8 \%$ & $8,9 \%$ & $0,0 \%$ & $0,0 \%$ & $0,1 \%$ & $2,2 \%$ & $0,5 \%$ \\
\hline Sa & $0,0 \%$ & $14,8 \%$ & $8,0 \%$ & $0,0 \%$ & $0,0 \%$ & $0,0 \%$ & $0,0 \%$ & $1,1 \%$ \\
\hline St & $0,0 \%$ & $15,5 \%$ & $7,1 \%$ & $0,0 \%$ & $0,0 \%$ & $0,0 \%$ & $0,5 \%$ & $0,6 \%$ \\
\hline T & $0,1 \%$ & $10,9 \%$ & $7,7 \%$ & $0,0 \%$ & $0,8 \%$ & $0,2 \%$ & $1,7 \%$ & $0,7 \%$ \\
\hline V & $0,5 \%$ & $4,8 \%$ & $10,9 \%$ & $0,5 \%$ & $1,0 \%$ & $0,3 \%$ & $4,2 \%$ & $1,2 \%$ \\
\hline $\mathrm{W}$ & $6,4 \%$ & $7,1 \%$ & $4,6 \%$ & $0,0 \%$ & $0,0 \%$ & $0,0 \%$ & $0,0 \%$ & $3,8 \%$ \\
\hline
\end{tabular}




\subsubsection{Grüne-Wähler von 1999}

\begin{tabular}{|c|c|c|c|c|c|c|c|c|}
\hline & SPÖ & ÖVP & FPÖ & GR & LIF & KPÖ & NW+So & Schw. \\
\hline B & $0,0 \%$ & $0,3 \%$ & $0,0 \%$ & $2,9 \%$ & $0,0 \%$ & $0,0 \%$ & $0,0 \%$ & $0,8 \%$ \\
\hline K & $0,0 \%$ & $1,8 \%$ & $0,0 \%$ & $2,6 \%$ & $0,0 \%$ & $0,0 \%$ & $0,0 \%$ & $0,6 \%$ \\
\hline N & $0,0 \%$ & $1,5 \%$ & $0,0 \%$ & $3,5 \%$ & $0,0 \%$ & $0,0 \%$ & $0,0 \%$ & $0,4 \%$ \\
\hline O & $0,1 \%$ & $1,1 \%$ & $0,0 \%$ & $4,9 \%$ & $0,1 \%$ & $0,0 \%$ & $0,0 \%$ & $0,5 \%$ \\
\hline Sa & $0,3 \%$ & $2,1 \%$ & $0,0 \%$ & $4,1 \%$ & $0,0 \%$ & $0,0 \%$ & $0,0 \%$ & $1,1 \%$ \\
\hline St & $0,0 \%$ & $0,2 \%$ & $0,0 \%$ & $3,6 \%$ & $0,1 \%$ & $0,2 \%$ & $0,5 \%$ & $0,5 \%$ \\
\hline T & $1,8 \%$ & $0,0 \%$ & $0,0 \%$ & $5,7 \%$ & $0,0 \%$ & $0,0 \%$ & $0,0 \%$ & $0,8 \%$ \\
\hline V & $0,3 \%$ & $0,0 \%$ & $0,0 \%$ & $7,1 \%$ & $0,0 \%$ & $0,0 \%$ & $0,0 \%$ & $1,3 \%$ \\
\hline W & $0,0 \%$ & $0,0 \%$ & $0,0 \%$ & $7,5 \%$ & $0,0 \%$ & $0,0 \%$ & $0,0 \%$ & $1,6 \%$ \\
\hline
\end{tabular}

\subsubsection{LIF-Wähler von 1999}

\begin{tabular}{|c|c|c|c|c|c|c|c|c|}
\hline & SPÖ & ÖVP & FPÖ & GR & LIF & KPÖ & NW+So & Schw. \\
\hline B & $0,4 \%$ & $0,5 \%$ & $0,0 \%$ & $0,7 \%$ & $0,0 \%$ & $0,0 \%$ & $0,0 \%$ & $0,7 \%$ \\
\hline K & $0,1 \%$ & $0,2 \%$ & $0,0 \%$ & $1,5 \%$ & $0,0 \%$ & $0,0 \%$ & $0,3 \%$ & $0,3 \%$ \\
\hline N & $0,0 \%$ & $0,2 \%$ & $0,0 \%$ & $2,2 \%$ & $0,1 \%$ & $0,0 \%$ & $0,0 \%$ & $0,3 \%$ \\
\hline $\mathrm{O}$ & $0,7 \%$ & $0,0 \%$ & $0,0 \%$ & $1,5 \%$ & $0,0 \%$ & $0,0 \%$ & $0,0 \%$ & $0,4 \%$ \\
\hline Sa & $0,0 \%$ & $0,0 \%$ & $0,0 \%$ & $3,0 \%$ & $0,0 \%$ & $0,0 \%$ & $0,0 \%$ & $1,1 \%$ \\
\hline St & $0,0 \%$ & $0,0 \%$ & $0,0 \%$ & $2,1 \%$ & $0,0 \%$ & $0,0 \%$ & $0,0 \%$ & $0,4 \%$ \\
\hline T & $0,0 \%$ & $0,0 \%$ & $0,0 \%$ & $2,9 \%$ & $0,0 \%$ & $0,0 \%$ & $0,0 \%$ & $0,7 \%$ \\
\hline $\mathrm{V}$ & $1,4 \%$ & $0,0 \%$ & $0,0 \%$ & $2,1 \%$ & $0,0 \%$ & $0,0 \%$ & $0,0 \%$ & $1,4 \%$ \\
\hline $\mathrm{W}$ & $0,0 \%$ & $2,1 \%$ & $0,0 \%$ & $3,0 \%$ & $0,0 \%$ & $0,0 \%$ & $0,0 \%$ & $1,7 \%$ \\
\hline
\end{tabular}

\subsubsection{Nichtwähler und sonstige Wähler von 1999}

\begin{tabular}{|c|c|c|c|c|c|c|c|c|}
\hline & SPÖ & ÖVP & FPÖ & GR & LIF & KPÖ & NW+So & Schw. \\
\hline B & $3,1 \%$ & $1,4 \%$ & $0,0 \%$ & $0,0 \%$ & $0,0 \%$ & $0,0 \%$ & $10,1 \%$ & $1,1 \%$ \\
\hline K & $2,1 \%$ & $2,6 \%$ & $0,0 \%$ & $0,6 \%$ & $0,4 \%$ & $0,1 \%$ & $16,2 \%$ & $1,1 \%$ \\
\hline $\mathrm{N}$ & $3,3 \%$ & $0,7 \%$ & $0,5 \%$ & $0,5 \%$ & $0,5 \%$ & $0,2 \%$ & $11,6 \%$ & $0,5 \%$ \\
\hline $\mathrm{O}$ & $3,2 \%$ & $0,7 \%$ & $0,0 \%$ & $0,8 \%$ & $0,5 \%$ & $0,3 \%$ & $13,7 \%$ & $0,5 \%$ \\
\hline $\mathrm{Sa}$ & $2,3 \%$ & $0,0 \%$ & $0,8 \%$ & $1,1 \%$ & $0,7 \%$ & $0,3 \%$ & $18,3 \%$ & $1,0 \%$ \\
\hline $\mathrm{St}$ & $3,4 \%$ & $0,0 \%$ & $0,7 \%$ & $0,0 \%$ & $0,5 \%$ & $0,5 \%$ & $17,0 \%$ & $0,5 \%$ \\
\hline $\mathrm{T}$ & $0,5 \%$ & $5,3 \%$ & $0,4 \%$ & $0,7 \%$ & $0,4 \%$ & $0,2 \%$ & $17,9 \%$ & $0,7 \%$ \\
\hline $\mathrm{V}$ & $1,2 \%$ & $8,9 \%$ & $0,0 \%$ & $1,9 \%$ & $0,3 \%$ & $0,0 \%$ & $15,3 \%$ & $1,6 \%$ \\
\hline $\mathrm{W}$ & $0,6 \%$ & $2,0 \%$ & $1,3 \%$ & $1,0 \%$ & $0,7 \%$ & $0,4 \%$ & $23,7 \%$ & $0,3 \%$ \\
\hline
\end{tabular}

\section{Methodische Bemerkungen}

Die vorliegende Wählerstromanalyse berechnet Schätzwerte für den Umfang der Wählerbewegungen zwischen den einzelnen Parteien. Sie verwendet dazu die Wahlergebnisse aller Gemeinden. Zunächst eine methodische Erläuterung am Beispiel des Vergleichs von NRW 99 und der NRW 02: Grundlage der verwendeten Methode ist die Annahme, dass sich die Wähler einer bestimmten Partei von 1999 diesmal (also 2002) in allen Gemeinden 
eines Bundeslandes nach etwa demselben Verteilungsschlüssel auf die 2002 kandidierenden Parteien aufgeteilt haben, dass also beispielsweise der Anteil aller SPÖ-Wähler von 1999, die 2002 die ÖVP gewählt haben, in allen Gemeinden annähernd gleich war. Ohne eine solche oder ähnliche Annahmen sind Wählerstromanalysen nicht möglich.

Details zum verwendeten Verfahren finden sich in Neuwirth (1984).

Für die politische Interpretation der Ergebnisse ist die Angabe von Schwankungsbreiten von hoher Bedeutung, weil Wählerbewegungen, deren Umfang mit hoher statistischer Absicherung angegeben werden kann, andere Schlussfolgerungen zulassen als mit hohen Unsicherheiten behaftete Schätzungen.

\section{Verfahrensfragen}

Bei Wählerstromanalysen gibt es drei Hauptprobleme technischer Natur:

Man muss eine eigene zusätzliche „Partei der Nichtwähler“ einführen, weil das Nichtwählen eine mögliche zu berücksichtigende Wählerentscheidung ist. Daher muss man auch Wählerströme von und zu dieser Gruppe berechnen und diese natürlich auch in der Analyse ausweisen. Selbstverständlich kann eine Wählerstromanalyse nur Auskunft über die Neuaufteilung der Wähler jener Parteien geben, die bei der betrachteten Vorwahl tatsächlich kandidiert haben.

Eigentlich geht das mathematische Modell der Wählerstromanalyse von der Fiktion der gleichen Wählerschaft bei beiden untersuchten Wahlen aus. Das entspricht natürlich nicht ganz der Wirklichkeit. Prinzipiell wäre es auch möglich, diese Änderung der Wählerschaft im Modell der Analyse zu berücksichtigen, allerdings stehen die dafür notwendigen Daten nicht zur Verfügung. Daher müssen wir uns mit einer Fiktion behelfen. Wir gehen von einer fiktiven Vorwahl aus, die etwa am Tage vor der aktuellen Wahl stattgefunden haben könnte, und bei der schon alle Wähler der neuen Wahl teilgenommen haben. Das (fiktive) Ergebnis dieser Wahl setzen wir so an, dass die Parteien dabei dieselben Anteile erreichen wie bei der realen Vorwahl. Das bedeutet ungefähr, dass wir zunächst einmal annehmen, dass die weggestorbenen Wähler und die Erstwähler bezogen auf die kandidierenden Parteien der Vorwahl etwa dieselbe Zusammensetzung aufweisen wie jene Wähler, die tatsächlich bei beiden Wahlen teilgenommen haben. Die berechneten Wählerströme beziehen sich dann auf den Vergleich der fiktiven Vorwahl mit der aktuellen Wahl. Hätten die weggestorbenen Wähler in höherem Maße für Partei A gestimmt als die restlichen Wähler und die hinzugekommenen Erstwähler in höherem Maß für Partei B, dann würde sich das in der statistischen Analyse als Wählerstrom von Partei A zu Partei B äußern.

Statistische Methoden liefern bei sehr kleinen Parteien keine zuverlässigen und aussagekräftigen Schätzungen mehr. Daher werden in der vorliegenden Analyse nicht alle kandidierenden Parteien untersucht, sondern nur jene Parteien, die 2002 bundesweit kandidiert haben. Die Stimmen der restlichen Kleinstparteien werden mit den Nichtund Ungültig-Wählern zusammengefasst. Jene Gruppe, die mit der Abkürzung NW+So bezeichnen wird, besteht also aus den Nichtwählern, den Ungültig-Wählern und den Wählern der in der Analyse nicht gesondert ausgewiesenen Kleinstparteien. 


\section{Literatur}

Erich Neuwirth. Schätzung von Wählerübergangswahrscheinlichkeiten. In Manfred Holler, editor, Wahlanalyse, tuduv-Verlag, München, 1984.

Adresse des Autors:

Ao.Univ.-Prof. Dr. Erich Neuwirth

Institut für Statistik und Decision Support Systems

Universität Wien

Universitätsstr. 5

A-1010 Wien

Tel. +4314277 / 38624

Fax +4314277/9386

E-mail: Erich.Neuwirth@univie.ac.at 